\title{
Indicador de falhas de qualidade baseado na percepção dos usuários de Habitação de Interesse Social
}

\author{
Indicator of quality failures based on the perception of \\ end users of Social Housing
}

\section{Letícia Ramos Berr \\ Márcia Elisa Soares Echeveste \\ Luciani Somensi Lorenzi \\ Carlos Torres Formoso}

\section{Resumo}

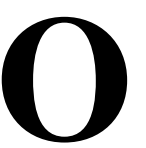

déficit habitacional é um grande desafio das políticas públicas no Brasil, não somente no sentido de suprir habitações na quantidade necessária, como também quanto à confiabilidade dos produtos ao longo da vida útil. Estudos prévios identificaram problemas de qualidade em empreendimentos de habitação de interesse social, que podem estar relacionados ao processo de desenvolvimento do produto ou a falhas na execução. Contudo, nenhum destes estudos avalia a qualidade das edificações de forma multidimensional, tampouco vincula explicitamente a percepção do usuário final do produto a outras dimensões da qualidade. Este artigo propõe um instrumento para mensurar a qualidade do produto, com base na percepção do usuário final das habitações de interesse social, cujo produto final é um conjunto de medidas relacionadas à percepção de qualidade dos usuários finais sobre a confiabilidade do produto habitacional. As medidas são obtidas por meio de uma pesquisa utilizando a técnica estatística de fração atribuível populacional (FAP). Propõe-se a aplicação do instrumento para identificar como as falhas nas edificações afetam a qualidade das habitações na visão do usuário. O entendimento desta relação pode contribuir para a melhoria de empreendimentos habitacionais e do controle de obras pela incorporação da visão do usuário final em indicadores de qualidade, os quais podem ser utilizados em sistemas de benchmarking.

Palavras-chave: Fração atribuível populacional. Habitação de Interesse Social. Indicador de falhas. Melhoria da qualidade. Qualidade percebida.

Letícia Ramos Berr Universidade Federal do Rio Grande do Sul Porto Alegre - RS - Brasil

Márcia Elisa Soares Echeveste Universidade Federal do Rio Grande do Sul Porto Alegre - RS - Brasil

Luciani Somensi Lorenzi Universidade Federal do Rio Grande do Sul Porto Alegre - RS - Brasil

Carlos Torres Formoso Universidade Federal do Rio Grande do Sul Porto Alegre - RS - Brasil

Recebido em 09/02/15 Aceito em 17/05/15

\section{Abstract}

Housing shortage is a major public policy challenge in Brazil, not only in terms of the need to supply the necessary number of dwellings, but also with regards to product reliability throughout the lifecycle. Previous studies have identified quality problems in social housing projects that may be related to the product development process as well as to production failures. However, none of those studies has assessed the quality of buildings in a multidimensional way, neither established a clear connection between the perception of end users and other quality dimensions. This paper proposes a tool to measure the quality of the products, based on the perception of end users of social housing projects, which outcome is a set of measures concerned with the perception of quality by the final users in relation to the reliability of the housing product. These measures are obtained through a survey, using a statistical technique named population attributable fraction $(P A F)$. This study proposes the application of the tool to identify how building defects can affect the quality of housing throughout the lifecycle period from the user's point of view. Understanding this relationship can contribute to the improvement of housing projects and production control by incorporating the perception of final users in quality metrics, which can be used in benchmarking systems.

Keywords: Defect indicator. Population attributable fraction. Quality improvement. Quality perception. Social Housing.

BERR, L. R.; ECHEVESTE, M. E. S.; LORENZI, L. S.; FORMOSO, C. T. Indicador de falhas de qualidade na percepção dos 19 usuários de Habitação de Interesse Social. Ambiente Construído, Porto Alegre,v. 15, n. 4, p. 19-35, out./dez. 2015. ISSN 1678-8621 Associação Nacional de Tecnologia do Ambiente Construído. http://dx.doi.org/10.1590/s1678-86212015000400037 


\section{Introdução}

Embora existam no país diversas ações no sentido de melhorar a qualidade da habitação de interesse social no Brasil, tais como os programas de gestão da qualidade nas empresas construtoras, o Programa Brasileiro de Qualidade e Produtividade no Habitat (PBQP-H) e a recente aprovação da Norma de Desempenho de Edificações (ABNT, 2013), há ainda muitos problemas de qualidade nesses empreendimentos. Esses problemas têm sido evidenciados por estudos sobre a qualidade de processos construtivos (BERR; FORMOSO, 2012), manifestações patológicas (ALEXANDRE, 2008; ANDRADE; DAL MOLIN, 1998; FIESS et al., 2004; RICHTER, 2007); avaliações pósocupação baseadas na percepção do usuário (ABIKO; ORNSTEIN, 2002; BONATTO et al., 2009; KOWALTOWSKI et al., 2004), e a própria avaliação de eficácia de sistemas de qualidade (BARTZ, 2007; CARDOSO, 2003).

De fato, existem diferentes perspectivas em relação às quais a qualidade de um produto pode ser avaliada. Garvin (2002), em seu trabalho seminal, sugere que a qualidade pode ser analisada por múltiplos critérios com base em diferentes abordagens, as quais são categorizadas como transcendental, cliente, produto, processo e valor. Essas abordagens são tratadas em uma segunda linha de critérios denominada por Garvin (1987) de dimensões: conformidade, confiabilidade, atendimento, qualidade percebida, desempenho, estética e durabilidade. O mesmo autor afirma que é improvável uma organização ter um bom desempenho em relação a todas as dimensões da qualidade: o sucesso de um produto no mercado depende normalmente de um pequeno número de dimensões. Entretanto, Garvin (1987) não limita a avaliação da qualidade a essas dimensões, por considerar que diferentes necessidades de avaliação são requeridas por diferentes produtos.

A maioria das avaliações da qualidade de empreendimentos habitacionais, como aquelas citadas acima, é realizada sob uma dimensão específica. De fato, a literatura aponta que estudos sobre qualidade percebida, em função de sua subjetividade, vem sendo tratados de forma dissociada de estudos que abordam outras dimensões da qualidade (PARASURAMAN, 1997; STONE-ROMERO; STONE; GREWAL, 1997; WOODRUFF, 1997; ZHANG, 2001). O mesmo é apontado para estudos relacionados a reclamações dos clientes (STONE-ROMERO; STONE; GREWAL, 1997), em que pese à forte relação entre reclamações dos consumidores e o real desempenho das habitações ao longo da vida útil. Assim, parece existir uma lacuna de conhecimento relacionada à necessidade de se avaliar a qualidade das edificações de forma multidimensional, vinculando a percepção do usuário final do produto a outras dimensões da qualidade. A adoção dessa abordagem multidimensional permitiria entender o efeito combinado de duas ou mais dimensões da qualidade na geração de valor.

O presente trabalho propõe um instrumento para mensurar a qualidade do produto, considerando duas dimensões da qualidade, a qualidade percebida e a confiabilidade do produto. $\mathrm{O}$ produto final desse instrumento é um indicador de falhas, que indica a percepção da qualidade por parte do usuário final das habitações de interesse social em relação à confiabilidade do produto habitacional entregue. Esse indicador pode retroalimentar as etapas de projetos e construção, assim como ser utilizado em sistemas de benchmarking.

Esse indicador é gerado com uma survey, estruturada a partir de uma base de dados de reclamações dos usuários HIS, sendo os dados produzidos analisados através da técnica da fração atribuível populacional (FAP) (HANLEY, 2001). Essa ferramenta foi testada em três empreendimentos habitacionais de interesse social da Região Metropolitana de Porto Alegre, no Rio Grande do Sul.

\section{Conceitos e dimensões da qualidade}

A proposta de Garvin (2002) de estruturar a qualidade em oito dimensões contempla as possibilidades de análises e avaliações usuais, mas essas não podem ser entendidas como uma rígida estrutura de avaliação. $\mathrm{O}$ autor aborda a existência de lacunas e sobreposições entre dimensões, o que pode indicar a possibilidade de variações conceituais das dimensões, bem como a existência de outras, a depender do processo ou produto em avaliação. Assim, neste tópico são apresentadas quatro das dimensões propostas por Garvin (2002), considerando a avaliação do produto habitação: conformidade, confiabilidade, qualidade percebida e desempenho. No segmento da construção de HIS essas dimensões são empregadas na avaliação da qualidade sem associação entre elas. Este trabalho aborda a possibilidade de obtenção de informações sobre a confiabilidade a partir da qualidade percebida e apresentar a visão do usuário sobre a qualidade da habitação em uso. Tanto a conformidade quanto o desempenho são discutidos devido às sobreposições com a dimensão da confiabilidade, e também pela relação de causa e 
efeito sobre a qualidade da habitação, mesmo com a grande distância temporal entre a ocorrência e a detecção de possíveis falhas.

\section{Conformidade}

Para Arditi e Gunaydin (1997), a conformidade está fortemente associada à definição de qualidade na construção civil e depende de que projetos e especificações sejam claros, concisos e uniformes, bem como devem possuir características que facilitem o processo de construção. Essa afirmação deixa clara a relação dependente da conformidade do produto na etapa de produção com a etapa de projeto, podendo ser essas sequenciais ou simultâneas. Assim, pode-se dizer que existem duas linhas projetuais para o alcance da conformidade, uma relacionada com os requisitos do cliente e uma complementar, que torna possível construir o produto de acordo com os requisitos do cliente. Nesse sentido, caberia uma melhor interação entre projeto e produção ao entender que não se pode promover a qualidade sem explicitar claramente aos envolvidos o que ela significa dentro de seu contexto de atuação (JURAN, 1988; GARVIN, 1987).

\section{Confiabilidade}

De acordo com Garvin (2002), as medidas de confiabilidade refletem a probabilidade de mau funcionamento de um produto ou de ele falhar em determinado período, e a partir de taxas de falhas pode-se aferir se o produto é adequado ao uso esperado (SAVAGE; CARR, 2001). Para Wu et al. (2006), a consideração da confiabilidade na etapa de projeto e produção afeta os custos de ciclo de vida de um sistema, considerando a soma das estimativas de custo dos sistemas a partir de sua concepção até seu descarte. Assim, a confiabilidade torna-se mais importante para os consumidores à medida que os tempos de parada $\mathrm{e}$ manutenção vão ficando frequentes (GARVIN, 2002). Ainda, para Wu et al. (2006), falhas relacionadas a problemas de produção não solucionados antes da entrega ao cliente afetam a confiabilidade e a durabilidade, uma vez que o produto com ausência de conformidade ao longo do tempo receberá manutenção antes do tempo esperado, movendo negativamente o ponto de equilíbrio da curva de custos do empreendimento ao longo do tempo e consequentemente afetando o desempenho esperado do produto.

\section{Desempenho}

Segundo Blachère (1969), o conceito de desempenho está associado ao comportamento da habitação em uso. Desde seu surgimento, o conceito de desempenho tem sofrido mudanças e incorporado fatores contemporâneos. A ISO 6241 (1984) foi um marco importante para a aplicação do conceito de desempenho na concepção de edifícios, visto que conseguiu expressar as exigências dos usuários em termos de requisitos (LORENZI, 2013). A norma NBR 15575 (ABNT, 2013) define desempenho como o comportamento em uso de um edifício e seus subsistemas. Entretanto, alguns autores destacam que esse conceito pode ser ampliado por meio da agregação de termos como "atender à função ao qual foi projetado", "durante a vida útil", "nas condições de exposição" (BORGES, 2008; MITIDIERI; HELENE, 1998; MITIDIERI, 2007; SILVA, 2010). Para Lorenzi (2013), o desempenho de uma edificação está no equilíbrio entre o meio, a função e a solução construtiva durante a vida útil.

\section{Qualidade percebida}

Segundo Wankhade e Dabade (2006), a qualidade percebida é uma medida da qualidade alcançada por um produto na visão do usuário. Por ser mais relacionada ao entendimento pessoal e individual do consumidor ante o observado ou vivenciado (ZEITHAML, 1988), a qualidade percebida apresenta alto nível de abstração em relação a outras dimensões da qualidade (WANKHADE; DABADE, 2006; ZEITHAML, 1988; ZHANG, 2001). Assim, as medidas de qualidade devem se focar nas percepções dos consumidores, e não somente em reclamações sobre a qualidade dos produtos que são oferecidos pelos fabricantes, ou em medidas de qualidade do produto de natureza objetiva (STONE-ROMERO; STONE; GREWAL, 1997). Nesse sentido, cabe entender quais são as dimensões da qualidade que o consumidor considera na avaliação de determinado produto (STONE-ROMERO; STONE; GREWAL, 1997) e, a partir da identificação dessas dimensões, estruturar um sistema de avaliação da qualidade para as diferentes etapas da vida das edificações. No contexto da habitação de interesse social não há o ato da compra do produto (pesquisa, escolha e aquisição). Assim, entende-se a qualidade percebida de uma habitação como sendo construída durante a experiência do usuário com a habitação, conceitualmente mais alinhado à visão de Zeithaml (1988).

\section{Relações entre as dimensões}

De acordo com Garvin (2002), as medidas de confiabilidade exigem que o produto esteja sendo usado por algum tempo, o que, alinhado com a visão de qualidade percebida de Zeithaml (1988), pode auxiliar na obtenção de uma medida que 
considere essas duas dimensões. Para $\mathrm{Wu}$ et al. (2006), a confiabilidade é fundamental para a avaliação do desempenho dos sistemas de uma edificação, devido ao fato de esta possuir relações com dimensões a jusante e a montante na vida útil do produto. Uma relação que expõe tal afirmação trata de problemas de conformidade não solucionados da etapa de produção, que são identificados após a entrega ao cliente, fato que amplia as possibilidades de falhas e reduz a confiabilidade do produto. Nesse caso, o conceito de conformidade, representando a causa de determinado comportamento na etapa de uso, sobrepõe-se ao conceito de confiabilidade, o qual pode indicar uma tendência de comportamento do produto ao longo do tempo, ou seja, o desempenho. Essas interações entre dimensões podem fornecer uma integração assertiva para o entendimento da percepção dos usuários das HIS e não somente evidenciam a existência de sobreposições entre as dimensões como também a necessidade de entender a influência que uma impõe sobre a outra (GARVIN, 2002; ZHANG, 2001), bem como a influência dessas sobre a qualidade percebida pelo usuário.

\section{Falhas e efeito de falhas}

Diversos conceitos relacionados à falta de qualidade são discutidos na literatura, tais como não conformidade, falha e defeito. Contudo, verificam-se muitas ambiguidades e sobreposições entre eles. Para a NBR ISO 9000 (ABNT, 2008) uma não conformidade refere-se ao não atendimento de um requisito, enquanto defeito refere-se ao não atendimento de um requisito relacionado a um uso especificado ou esperado. Já a falha é conceituada como o afastamento das boas práticas devido a um erro previsto pelas margens de desvio especificadas, que pode ou não ser corrigida antes da entrega do edifício (RAUSAND; KNUT, 1996; ATKINSON, $1987^{1}$ apud MILLS; LOVE; WILLIAMS, 2009). No presente trabalho entende-se que as não conformidades observadas na etapa de uso de uma edificação podem ser tratadas como falhas, ao considerar que a observação ocorre na etapa de uso, sem a interrupção da utilização do imóvel. Por sua vez, os efeitos das falhas, segundo Helman e Andery (1995), referem-se às formas de como as falhas afetam o desempenho do produto do ponto de vista do cliente.

\section{Operacionalização de conceitos de qualidade na construção civil}

Por fim, os sistemas de qualidade que integrem medidas objetivas e subjetivas, vinculadas à produção e ao uso (HOPP; SPEARMAN, 2000; ZHANG, 2001), são úteis para todos os intervenientes. Quando se trata de um sistema de qualidade para a construção civil, entender as peculiaridades desse setor pode auxiliar na redução de impactos negativos que essas possam fornecer ao fluxo da construção (KOSKELA, 1992). Entre as peculiaridades a própria definição do produto habitação de interesse social dificulta uma avaliação clara de sua qualidade (ARDITI; GUNAYDIN, 1997).

\section{Método}

A estratégia de pesquisa empregada foi o desenvolvimento de uma survey com o propósito de obter dados para a formulação do indicador de falhas na visão dos usuários.

A Figura 1 apresenta o delineamento da pesquisa, dividida em três etapas. $\mathrm{Na}$ etapa 1 foi realizado um levantamento das falhas das edificações na etapa de uso a partir de estudos anteriores sobre reclamações de usuários e manifestações patológicas identificadas em empreendimentos habitacionais após a ocupação, tendo como produto final um diagrama de afinidades. Na etapa 2 foi proposto o indicador com o propósito de operacionalizar os conceitos de confiabilidade e qualidade percebida, as previsões de estratificação do indicador para análise, bem como a seleção dos métodos de análise e técnicas estatísticas para determinar as variáveis e escalas de medidas que serão empregadas. Também foi construído o instrumento de coleta a partir do diagrama de afinidades, realizado o planejamento amostral e feita a coleta dos dados. Os produtos da etapa 2 foram a definição da estrutura do indicador proposto, o método para obtê-lo e a base de dados sobre a qualidade das habitações na percepção dos usuários. Na etapa 3 foram realizadas análises comparativas entre as métricas obtidas.

\footnotetext{
${ }^{1}$ ATKINSON, G. A Century of Defects. Building, v. 252, p. 54-55, Jun. 1987.
} 
Figura 1 - Delineamento da pesquisa

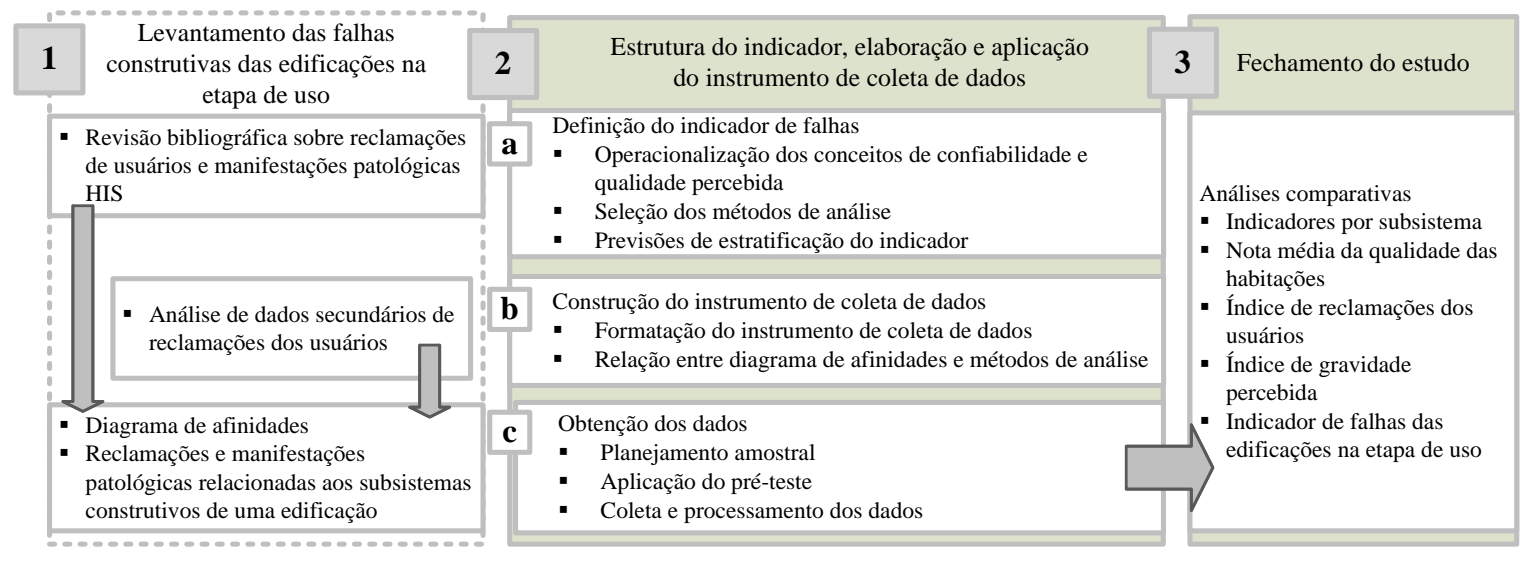

\section{Levantamento das falhas das edificações na etapa de uso}

A primeira etapa da pesquisa visou entender o padrão de falhas das habitações reclamadas pelos usuários. Assim, analisaram-se dados secundários (fonte não pública) contendo reclamações de usuários de HIS. Esses dados foram obtidos por uma administradora de imóveis, responsável pela gestão da operação e manutenção de empreendimentos do Programa de Arrendamento Residencial (PAR), a qual realizava atividades de reparo das edificações ou encaminhamento delas a outras instituições responsáveis. Também foram analisados trabalhos acadêmicos que avaliaram as reclamações de usuários e a qualidade construtiva das edificações, buscando identificar padrões das falhas, bem como outras falhas não reclamadas, mas que na visão técnica pudessem complementar as análises. Como produto desta fase os dados obtidos foram organizados na forma de um diagrama de afinidades que relaciona as reclamações dos usuários às partes de uma edificação: fundações, estruturas, vedações verticais, vedações horizontais, esquadrias, pintura e sistemas prediais. Essas partes referem-se aos subsistemas de uma edificação, categorizados com base na lista de serviços controlados do Programa Brasileiro de Qualidade e Produtividade no Habitat (PBQP-H), devido a seu amplo uso por empresas construtoras.

\section{Estrutura do indicador, elaboração e aplicação do instrumento de coleta de dados}

\section{Definição do indicador de falhas}

Para propor um instrumento de mensuração da qualidade na percepção dos usuários, buscou-se identificar o tipo de resposta que pudesse ser incorporado em rotinas de coleta, processamento e análise de dados das empresas construtoras, bem como pressupor a utilização de dados sobre reclamações de clientes cujas empresas com sistemas de gestão da qualidade dispõem. Por fim, a definiç̧ão da resposta com o formato de indicador possibilita a incorporação dessa informação aos sistemas de gestão da qualidade certificados.

Assim, para operacionalizar os conceitos de confiabilidade e qualidade percebida, foram estabelecidos dois objetivos secundários para nortear a construção dos indicadores:

(a) verificar a influência de cada item falho na percepção do usuário; e

(b) priorizar os sistemas construtivos segundo a percepção dos usuários.

Dessa forma, a técnicas estatísticas relacionam a declaração do beneficiário sobre a existência de um item falho, ou seja, se ele percebe que está exposto, com a influência e a gravidade que esta falha possui na percepção de qualidade de cada subsistema da edificação. Os demais objetivos secundários estão relacionados às respostas esperadas e à aplicação do instrumento de coleta. Todos são apresentados no Quadro 1 desdobrados nos métodos de análise para alcançá-los.

\section{Construção do instrumento de coleta de dados}

O instrumento de coleta de dados partiu da categorização da base de dados secundária, que apresentava 7.574 reclamações, das quais 4.300 foram classificadas como problemas construtivos. Essas reclamações foram agrupadas pelos subsistemas da edificação e pelas diferentes atividades da etapa de obras (serviços) vinculados a ela, originando um diagrama de afinidades. Um trecho do diagrama para o subsistema vedações verticais/paredes está apresentado no Quadro 1. 
A estrutura do instrumento de coleta atende a quatro elementos necessários parao atendimento aos objetivos da pesquisa:

(c) percepção de gravidade das falhas;

(d) percepção de qualidade da unidade habitacional como um todo;

(e) percepção de qualidade dos subsistemas construtivos da unidade habitacional; $\mathrm{e}$

(f) percepção de falhas na unidade habitacional.

Cada parte do questionário foi desenvolvida de forma a possibilitar o uso dos métodos de análise e técnicas estatísticas apresentadas no Quadro 2 e, assim, obter o indicador de falhas proposto.

No primeiro elemento do questionário empregouse a técnica do incidente crítico e de priorização das respostas para identificar a percepção do usuário sobre as falhas e sua gravidade. No segundo elemento, solicita-se que o usuário atribua uma nota para a qualidade de sua unidade habitacional em uma escala de dez pontos, de forma a possibilitar sua comparação com o indicador proposto ao final. A nota obtida serve como parâmetro de validação do instrumento de coleta, uma vez que se presume que a qualidade percebida pelo usuário seja inversamente proporcional ao indicador de falhas. O terceiro elemento do questionário visa identificar a qualidade percebida pelo usuário sobre cada um dos subsistemas da edificação, descritos no instrumento de coleta a partir de uma escala ordinal (1) Muito ruim, (2) Ruim, (3) Bom e (4) Muito bom. Contudo, para o emprego dos dados de qualidade dos subsistemas na técnica da FAP estes foram reescalonados para o nível binário (0)
Ruim e (1) Bom, devido ao pequeno tamanho da amostra para o emprego da escala completa. Por fim, no quarto elemento do questionário (Quadro 3) obtém-se as falhas construtivas categorizadas de acordo como diagrama de afinidades proposto. Como resposta, os usuários declaram a presença ou ausência - (1) ou (0) respectivamente - de determinada falha percebida em um padrão de respostas também binário.

\section{Obtenção dos dados}

\section{Plano amostral e a coleta de dados}

Os empreendimentos habitacionais de interesse social - A, B e C - selecionados para o estudo totalizam 512 unidades habitacionais (UH) e localizam-se na Região Metropolitana de Porto Alegre, no Rio Grande do Sul. Contudo, a população do estudo, dentro desses empreendimentos, foi delimitada pelos seguintes condicionantes:

(a) ser composto de unidades habitacionais pertencentes ao banco de dados de reclamações dos usuários;

(b) pertencer a uma mesma construtora;

(c) possuir entre um e dois anos de uso; e

(d) ter sido executado em alvenaria estrutural.

O primeiro critério foi empregado como forma de possibilitar a comparação entre o resultado do indicador de falhas com índices de reclamações disponíveis e, assim, colaborar na validação da capacidade do indicador de aferir. Dessa forma, 200 UH reclamantes formaram a população do estudo.

Quadro 1 - Diagrama de afinidades do subsistema vedações verticais

\begin{tabular}{|c|c|c|}
\hline SUBSISTEMA & SERVIÇO & PROBLEMAS RECLAMADOS NA ETAPA DE USO \\
\hline \multirow{12}{*}{$\begin{array}{l}\text { Vedações } \\
\text { Verticais/Paredes }\end{array}$} & \multirow{2}{*}{$\begin{array}{l}\text { Execução de alvenaria } \\
\text { não estrutural e de } \\
\text { divisória leve }\end{array}$} & Tijolos soltos de elementos secundários \\
\hline & & Fissura ou rachadura nas paredes de vedação \\
\hline & \multirow{8}{*}{$\begin{array}{l}\text { Execução de } \\
\text { revestimento das paredes }\end{array}$} & Fissura ou rachadura nas paredes \\
\hline & & Infiltração na parede \\
\hline & & Reboco caindo \\
\hline & & Ocorrência de mofo nas paredes \\
\hline & & Rejunte das cerâmicas solto ou inexistente \\
\hline & & Azulejo caindo, rachado ou quebrado \\
\hline & & Acabamento das paredes argamassadas ruim ou inexistente \\
\hline & & Presença de umidade \\
\hline & \multirow{2}{*}{ Pintura das paredes } & Existência de bolhas ou partes descascando \\
\hline & & Manchas ou falhas de cobrimento na pintura das paredes \\
\hline
\end{tabular}

24 Berr, L. R.; Echeveste, M. E. S.; Lorenzi, L. S.; Formoso, C. T. 
Quadro 2 - Relação entre os objetivos específicos e os métodos de análise de dados

\begin{tabular}{|l|l|}
\hline \multicolumn{2}{|c|}{ Definição do indicador de falhas e construção do instrumento de coleta de dados } \\
\hline Objetivos específicos & Método de análise \\
\hline $\begin{array}{l}\text { Verificar a influência de cada item falho na } \\
\text { percepção do usuário sobre a qualidade de um } \\
\text { subsistema construtivo. }\end{array}$ & Fração atribuível populacional. \\
\hline $\begin{array}{l}\text { Priorizar os subsistemas construtivos segundo a } \\
\text { percepção de gravidade do usuário. }\end{array}$ & $\begin{array}{l}\text { Técnica do incidente crítico. } \\
\text { Frequência de ocorrência. }\end{array}$ \\
\hline $\begin{array}{l}\text { Propor um indicador de falhas para cada um } \\
\text { dos subsistemas construtivos. }\end{array}$ & $\begin{array}{l}\text { Média ponderada pelas frações atribuíveis } \\
\text { populacionais das proporções de itens falhos. }\end{array}$ \\
\hline $\begin{array}{l}\text { Propor um indicador de falhas para cada } \\
\text { empreendimento. }\end{array}$ & $\begin{array}{l}\text { Média dos indicadores dos subsistemas de um } \\
\text { empreendimento ponderada pelos resultados da } \\
\text { técnica do incidente crítico. }\end{array}$ \\
\hline $\begin{array}{l}\text { Relacionar a nota da qualidade dada pelo } \\
\text { usuário com o indicador de falhas. }\end{array}$ & $\begin{array}{l}\text { Correlação entre a avaliação da qualidade feita pelo } \\
\text { usuário e o indicador de falhas. }\end{array}$ \\
\hline $\begin{array}{l}\text { Avaliar a aplicação do instrumento de coleta } \\
\text { com usuários e técnicos. }\end{array}$ & Pré-teste do instrumento de coleta. \\
\hline $\begin{array}{l}\text { Garantir a homogeneidade dos dados coletados } \\
\text { por diferentes pesquisadores. }\end{array}$ & Protocolo de coleta de dados. \\
\hline
\end{tabular}

\section{Quadro 3 - Trechos do quarto elemento do instrumento de coleta}

\begin{tabular}{|c|c|c|}
\hline 4.1. Com relação às PAREDES de seu apartamento: & $\operatorname{Sim}(1)$ & Não $(0)$ \\
\hline \multicolumn{3}{|l|}{ (a) Há rachaduras? } \\
\hline \multicolumn{3}{|l|}{ (b) Há infiltração, mofo ou umidade? } \\
\hline \multicolumn{3}{|l|}{ (c) Falta acabamento (reboco/azulejo/pintura) em alguma parede? } \\
\hline 4.2. Com relação ao PISO e ao TETO de seu apartamento: & $\operatorname{Sim}(1)$ & Não $(0)$ \\
\hline \multicolumn{3}{|l|}{ (a) Há rachaduras no teto? } \\
\hline \multicolumn{3}{|l|}{ (b) Há infiltração no teto? } \\
\hline \multicolumn{3}{|l|}{ (c) Há rachaduras no piso? } \\
\hline 4.3. Com relação às PORTAS e JANELAS de seu apartamento: & $\operatorname{Sim}(1)$ & Não $(0)$ \\
\hline \multicolumn{3}{|l|}{ (a) Há problemas na pintura e acabamento das portas e janelas? } \\
\hline \multicolumn{3}{|l|}{ (b) Dobradiças e/ou fechaduras estão com problemas? } \\
\hline \multicolumn{3}{|l|}{ (c) Há portas empenadas ou cedendo? } \\
\hline 4.4. Com relação à INSTALAÇÃO ELÉTRICA de seu apartamento: & $\operatorname{Sim}(1)$ & Não $(0)$ \\
\hline \multicolumn{3}{|l|}{ (a) Há ausência de algum ponto de luz, tomada ou outro acabamento elétrico? } \\
\hline \multicolumn{3}{|l|}{ (b) Há algum ponto de luz ou tomada que não funcione? } \\
\hline \multicolumn{3}{|l|}{ (c) Já houve algum curto-circuito ou incidência de choque? } \\
\hline 4.5. Com relação a INSTALAÇÕES HIDROSSANITÁRIAS de seu apartamento: & $\operatorname{Sim}(1)$ & Não (0) \\
\hline \multicolumn{3}{|l|}{ (a) Ao receber o imóvel, faltou pia, vaso, torneiras, registros? } \\
\hline \multicolumn{3}{|l|}{ (b) Ao receber o imóvel, a pia ou o vaso estavam quebrados ou rachados? } \\
\hline (c) Algum registro, descarga, torneira não funciona? & & \\
\hline
\end{tabular}


A coleta de dados foi realizada por uma equipe com formação em engenharia civil ou arquitetura ${ }^{1}$. Os membros da equipe envolveram-se no desenvolvimento do IC, e assim não houve a necessidade de treinamento prévio. No entanto, foi desenvolvido um protocolo de coleta para futuras coletas de dados como forma de buscar a homogeneidade delas.

\section{Amostragem}

Com base nas definições do plano amostral apresenta-se a população do estudo (Tabela 1). Na tabela são apresentados o tamanho de cada empreendimento, a idade dele quando o usuário reclamou sobre falhas construtivas e a idade quando os dados foram coletados para este trabalho. A amostra estabelecida guarda proporcionalidade aproximada de $30 \%$ entre o número de unidades reclamantes (UHR) e de $10 \%$ com o total de unidades do empreendimento.

O tamanho amostral foi calculado com base em proporção $(n=200 ; z=1,96 ; p=0,5 ; e=0,1)$, e a partir do tamanho amostral obtido os domicílios foram selecionados por amostragem aleatória simples. Assim, foram coletados dados em 66 unidades habitacionais reclamantes. Também foram estabelecidos critérios sistemáticos para o caso de alteração das unidades habitacionais sorteadas. No caso, escolhe-se a com número da $\mathrm{UH}$ imediatamente inferior ao sorteado de maneira sucessiva, até efetuar a entrevista.

\section{Pré-teste}

Foram realizados dois pré-testes, o primeiro para validação interna do IC na visão técnica, com a colaboração de especialistas que inicialmente responderam ao questionário da mesma forma que os usuários e que em seguida analisaram a estrutura dele. $\mathrm{O}$ segundo pré-teste foi realizado com uma parcela da população do estudo como forma de ajustar a linguagem do instrumento de coleta ao entendimento dos entrevistados. Assim, com base no pré-teste novos ajustes foram conduzidos, entre os quais estão a reformulação de perguntas por gerar respostas muito amplas e a complementação da explicação de alguns itens.

\section{Checagem do indicador}

Para a validação estatística do IC foi calculado o coeficiente de correlação de Pearson entre as notas da qualidade declaradas pelo respondente e os resultados do indicador de falhas. A hipótese dos pesquisadores é a de que há uma correlação inversa entre a nota do usuário e o resultado do indicador e que tal resultado indicaria coerência entre $\mathrm{o}$ indicador $\mathrm{e}$ as notas atribuídas pelos usuários.

\section{Processamento dos dados}

$\mathrm{Na}$ última fase, o tratamento dos dados foi estruturado para a obtenção do indicador de falhas proposto $\left(I_{\text {falhas }}\right)$ e os indicadores secundários: Indicador por Subsistema (IS), o Índice de Gravidade Percebida (GP). Para finalmente efetuar análises entre os referidos indicadores, comparativamente com a média da nota da qualidade da habitação atribuída pelo usuário durante a pesquisa, o número de reclamações por unidade habitacional, o percentual de unidades habitacionais reclamantes obtidos da base de dados secundários, fração atribuível das falhas pesquisadas e a proporção de falhas construtivas identificadas pelos usuários.

\section{Tabela 1 - População do estudo}

\begin{tabular}{|c|c|c|c|c|c|c|}
\hline \multirow[b]{2}{*}{ Empreendimento } & \multirow[b]{2}{*}{ Cidade } & \multicolumn{2}{|c|}{ ID - Idade do EHIS } & \multirow[b]{2}{*}{ UHR } & \multirow[b]{2}{*}{ UHA } & \multirow[b]{2}{*}{$\mathbf{U H}$} \\
\hline & & $\begin{array}{c}\text { Dados } \\
\text { secundários }\end{array}$ & $\begin{array}{l}\text { Coleta } \\
\text { dados }\end{array}$ & & & \\
\hline EHIS A & Novo Hamburgo & 19 meses & 44 meses & 46 & 15 & 160 \\
\hline EHIS B & Novo Hamburgo & 19 meses & 44 meses & 63 & 18 & 160 \\
\hline EHIS C & Sapiranga & 19 meses & 44 meses & 91 & 34 & 192 \\
\hline
\end{tabular}
Nota: Legenda:
ID - idade dos EHIS quando foram efetuadas as reclamações e a idade na época da coleta dos dados;
UHR - total de unidades habitacionais que efetuaram reclamações sobre problemas construtivos;
UHA - total de unidades habitacionais da amostra do estudo em cada empreendimento;
$\mathrm{UH}$ - total de unidades habitacionais em cada empreendimento; e
ID - idade dos EHIS quando foram efetuadas as reclamações e na época da coleta dos dados.

\footnotetext{
${ }^{2}$ Pesquisadores que participaram deste estudo: (equipe de engenharia) Carlos Torres Formoso; Camila Famá; Fernanda Sbaraini Bonatto; Juliana Moehlecke; Leticia Ramos Berr; (equipe de estatística) Márcia Echeveste; Jáderson Radaelli; Lisiane de Souza Nunes de Moura; Priscila Valandro Ferreira; Ubirajara Sampaio.
} 


\section{Resultados do estudo}

\section{Apresentação Indicador de Falhas e seus componentes}

Inicialmente é apresentada a estrutura de formulação do indicador de falhas por subsistema (IS), seguida da apresentação do índice de gravidade percebida (GP) e do indicador de falhas geral $\left(I_{\text {falhas }}\right)$.

Para cada subsistema pesquisado foi elaborado um indicador de falha específico, Equação 1, por meio da média das proporções de cada um dos itens falhos declarados, pelo número total de usuários que foram entrevistados. Essa proporção foi ponderada pela fração atribuível populacional (FAP) e varia entre 0 e 1 , sendo 0 a ausência falhas na percepção dos usuários.

$$
I S=\frac{\sum_{i=1}^{k} \operatorname{proporção~}_{i} F A P_{i}}{\sum_{j=1}^{n} F A P_{j}}
$$

Em que:

IS = indicador de falha por subsistema;

proporção = proporção de cada item falho no subsistema;

$\mathrm{FAP}_{\mathrm{i}}=$ fração atribuível populacional de determinado item falho;

$\mathrm{FAP}_{\mathrm{i}}=$ fração atribuível de determinado subsistema;

$\mathrm{k}=$ número de itens falhos avaliados dentro do subsistema em análise;

$\mathrm{i}=$ itens falhos; $\mathrm{e}$

$\mathrm{j}=$ subsistemas.

A fração atribuível populacional corresponde à proporção de usuários com percepção negativa da qualidade que poderia ser reduzida caso a exposição do usuário à falha fosse eliminada. Dessa forma, sua importância se deve ao fato de permitir quantificar o efeito que uma falha gera na visão do usuário (HANLEY, 2001). Com base nessa quantificação pode-se empregar uma estratégia preventiva ao se buscar reduzir a não conformidade ou falha na fase de construção de empreendimentos futuros. Assim, é possível também identificar os fatores que têm maior impacto na visão dos usuários, possibilitando a priorização no tratamento deles.

O índice de gravidade percebida (GP) do j-ésimo subsistema (Eq. 2) é uma ponderação obtida por meio da priorização dos subsistemas de acordo com a gravidade apontada pelos usuários pela técnica do incidente crítico. Nesse caso, o subsistema contendo mais falhas consideradas graves recebe maior peso.

$G P_{j}=3 p_{1 j}+2 p_{2 j}+p_{3 j}$

Em que:

$\mathrm{p}_{1 \mathrm{j}}$ é a frequência relativa do subsistema $j$ na classificação "mais grave";

$\mathrm{p}_{2 \mathrm{j}}$ é a frequência relativa do subsistema $j$ na classificação "medianamente grave"; e

$\mathrm{p}_{3 \mathrm{j}}$ é a frequência relativa do subsistema $j$ na classificação "menos grave".

Por fim, o cálculo do indicador de falhas geral $\left(I_{\text {Falhas }}\right.$ ) (Eq. 3) é o resultado do somatório dos IS ponderados pela GP. Assim, o indicador de falhas geral, mesmo de forma agregada, pode ser entendido como a percepção dos usuários sobre a confiabilidade geral do empreendimento.

$I_{\text {Falhas }}=\frac{\sum_{j=1}^{n} I S_{j} G P_{j}}{\sum_{j=1}^{n} G P_{j}}$

Eq. 3

\section{Indicador por subsistema: resultados}

Como forma de melhorar o entendimento do indicador proposto são apresentados a seguir os passos de seu cálculo com base nos dados coletados.

\section{Fração atribuível populacional e proporção de itens falhos}

Um exemplo de cálculo da fração atribuível populacional (FAP) é apresentado na Tabela 2 (Eq. 4), a partir de uma tabela de contingência bivariada para o item falho reclamado "rachaduras" com relação ao subsistema vedações verticais "paredes".

Dessa forma cada item do questionário, baseado em reclamações sistêmicas dos empreendimentos, busca identificar em cada unidade habitacional se o usuário percebe a existência da falha e a relaciona com a percepção de qualidade do subsistema ao qual a falha pertence.

FAP $=\frac{\frac{43}{67}-\frac{10}{19}}{\frac{43}{67}}=0,179$

Eq. 4

Como resultado do cálculo da FAP obtém-se o impacto do item na percepção da qualidade da edificação por parte do usuário, ou seja, se o item fosse eliminado o usuário teria sua percepção negativa reduzida em $17,9 \%$ com relação a todo o 
subsistema. A Tabela 3 apresenta as FAP das falhas pesquisadas.

No mesmo sentido, a falha "infiltração" do subsistema "paredes" apresenta maior impacto na percepção negativa da qualidade por parte do usuário (48\%); nesse caso apenas 4 usuários declararam não perceber a falha, e 42 declararam que a qualidade do subsistema é ruim. Assim, sua eliminação pode contribuir mais fortemente na percepção do usuário sobre a qualidade do subsistema.

Também são calculadas as proporções de cada item falho no referido subsistema. Essa medida é obtida a partir do número de vezes, em média, que um item falho foi declarado pelos entrevistados. De maneira geral (Tabela 3) o subsistema "paredes" apresenta elevadas proporções.

Tabela 2 - Tabela de contingência bivariada - item rachaduras no subsistema paredes

\begin{tabular}{|c|c|c|c|c|}
\hline & \multicolumn{3}{|c|}{ Percepção de qualidade do subsistema vedação vertical/paredes } & \multirow[t]{2}{*}{ Total } \\
\hline & & Bom & Ruim & \\
\hline \multirow{3}{*}{ Há rachaduras? } & Não & 9 & 10 & 19 \\
\hline & Sim & 15 & 33 & 48 \\
\hline & Total & 24 & 43 & 67 \\
\hline
\end{tabular}

Tabela 3 - Fração atribuível populacional dos itens pesquisados em cada subsistema

\begin{tabular}{|c|c|c|}
\hline & Item não conforme & FAP \\
\hline \multirow{8}{*}{ 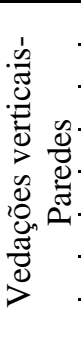 } & Rachaduras nas paredes & 0,179 \\
\hline & Infiltração, mofo, umidade & 0,481 \\
\hline & Ausência de acabamento & 0,008 \\
\hline & Irregularidades no reboco & 0,142 \\
\hline & Reboco caindo ou quebrado & 0,172 \\
\hline & Azulejos soltos, sem rejuntes, faltam peças & 0,013 \\
\hline & Bolhas ou descascados & 0,169 \\
\hline & Manchas ou falhas na pintura & 0,050 \\
\hline \multirow{8}{*}{ 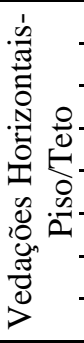 } & Rachaduras no teto & 0,241 \\
\hline & Infiltração no teto & 0,202 \\
\hline & Rachaduras no piso & 0,427 \\
\hline & O piso está solto, sem rejunte ou faltam peças & 0,111 \\
\hline & Piso irregular & 0,345 \\
\hline & Há manchas no piso & 0,110 \\
\hline & Na área de serviço ou box a água empoça & 0,042 \\
\hline & Infiltração no piso & 0,077 \\
\hline \multirow{4}{*}{ 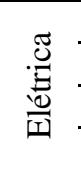 } & Falta ponto de luz, tomada ou acabamento elétrico & 0,118 \\
\hline & Ponto de luz ou tomada que não funciona & 0,189 \\
\hline & Ocorrência de curto-circuito ou incidência de choque & 0,162 \\
\hline & Queda de disjuntor no uso do chuveiro & 0,070 \\
\hline \multirow{4}{*}{ 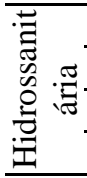 } & No recebimento do imóvel, faltou pia, vaso, torneiras, registros & 0,072 \\
\hline & No recebimento do imóvel, a pia ou o vaso estavam quebrados ou rachados & 0,063 \\
\hline & Algum registro, descarga, torneiras não funcionam & 0,305 \\
\hline & Há algum problema de vazamento & 0,080 \\
\hline \multirow{8}{*}{ 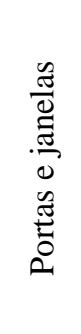 } & Há problemas na pintura e acabamento & 0,206 \\
\hline & Dobradiças e/ou fechaduras com problemas & 0,085 \\
\hline & Há portas empenadas ou cedendo & 0,059 \\
\hline & Há portas com frestas ou rachadas & 0,056 \\
\hline & Porta abrindo no sentido contrário & 0,016 \\
\hline & Entra água pelas janelas & 0,077 \\
\hline & Há janelas empenadas ou cedendo & 0,134 \\
\hline & Há janelas com frestas ou rachadas & 0,021 \\
\hline
\end{tabular}

28 Berr, L. R.; Echeveste, M. E. S.; Lorenzi, L. S.; Formoso, C. T. 
Tabela 4 - Proporções de itens falhos no subsistema de vedações verticais/paredes

\begin{tabular}{lcccc}
\hline & Geral & EHISA & EHISB & EHISC \\
\hline Rachaduras nas paredes & 0,72 & 0,60 & 0,67 & 0,79 \\
Infiltração, mofo, umidade & 0,96 & 0,87 & 0,94 & 1,00 \\
Ausência de acabamento & 0,34 & 0,53 & 0,33 & 0,26 \\
Irregularidades no reboco & 0,48 & 0,67 & 0,29 & 0,50 \\
Reboco caindo ou quebrado & 0,34 & 0,43 & 0,35 & 0,29 \\
Azulejos soltos, sem rejuntes, faltam peças & 0,10 & 0,27 & 0,17 & 0,00 \\
Bolhas ou descascados & 0,55 & 0,40 & 0,44 & 0,68 \\
Manchas ou falhas na pintura & 0,31 & 0,33 & 0,22 & 0,35 \\
\hline
\end{tabular}

Com base na Tabela 4 destaca-se a falha de infiltração, mofo e umidade $(\mathrm{p}=0,96)$ e ocorrências de bolhas ou descascando $(p=0,72)$. Esses itens são críticos para a percepção de qualidade do usuário, pois indicam que foram observados $96 \%$ e $72 \%$ das vezes respectivamente.

Também se observou o comportamento da FAP comparativamente à frequência de ocorrência das falhas obtidas, segundo os dados registrados como reclamações na empresa em fonte de dados secundários. Na Figura 2 são apresentados os valores das duas medidas estatísticas empregadas para os itens que compõem a avaliação do subsistema vedações verticais "paredes". Os itens são:

(a) rachaduras;

(b) infiltração, mofo, umidade;

(c) ausência de acabamento;

(d) irregularidades no reboco;

(e) reboco caindo ou quebrado;

(f) azulejos soltos, sem rejuntes, falta de peças;

(g) bolhas ou descascando; e

(h) manchas ou falhas na pintura.

O comportamento da fração atribuível mostra que alguns itens têm mais influência que outros na percepção da qualidade pelo usuário, apesar de nem sempre apresentarem a maior frequência de ocorrência, e a relação inversa também ocorre.

Como exemplo, na Figura 2, percebe-se que os itens "c - falta de acabamento" e "e - reboco caindo ou quebrado" possuem frequências de ocorrência semelhantes, no entanto o item "e" influencia mais na percepção de qualidade do usuário sobre o subsistema paredes. Outra relação pode ser verificada com os itens "a - rachaduras", "e - reboco caindo ou quebrado" e "g - existência de bolhas ou descascando", que apresentam FAP semelhantes e diferentes frequências de ocorrência, indicando que mesmo com um menor número de reclamações o item "e" tem a mesma influência na percepção do usuário que o item "a" ou o "g".

\section{Resultados do indicador por subsistema (IS)}

Para a formulação do indicador por subsistema de vedações verticais/paredes são empregados os dados apresentados na Tabela 3 e na Tabela 4. O valor das proporções é multiplicado pelo valor das frações atribuíveis populacionais de cada item pesquisado, os quais são somados e divididos pelo somatório das FAP do subsistema. A Tabela 5 apresenta esses valores e a Equação 5 apresenta o resultado do indicador do subsistema vedações verticais/paredes.

$I S_{\text {paredes }}=\frac{0,830}{1,214}=0,684$

Os indicadores dos subsistemas obtidos neste estudo são apresentados na Tabela 6, utilizando a Equação 1. A referida tabela apresenta a comparação entre os empreendimentos e uma avaliação geral de cada subsistema.

O valor representa o quanto uma ação em determinado subsistema poderia impactar na melhoria da percepção do usuário sobre a qualidade construtiva da edificação. Ao analisar esses indicadores, é possível inferir que os usuários possuem maior percepção negativa da qualidade do subsistema paredes, por apresentar o maior índice de falhas no referido subsistema $(\mathrm{IS}=0,68)$. No caso das falhas em instalações elétricas (IS=0,20), o impacto na percepção de qualidade da habitação é o menor. 
Figura 2 - Frequência de ocorrência de falha declarada e fração atribuível populacional (FAP) dos itens não conformes do subsistema vedações verticais/paredes

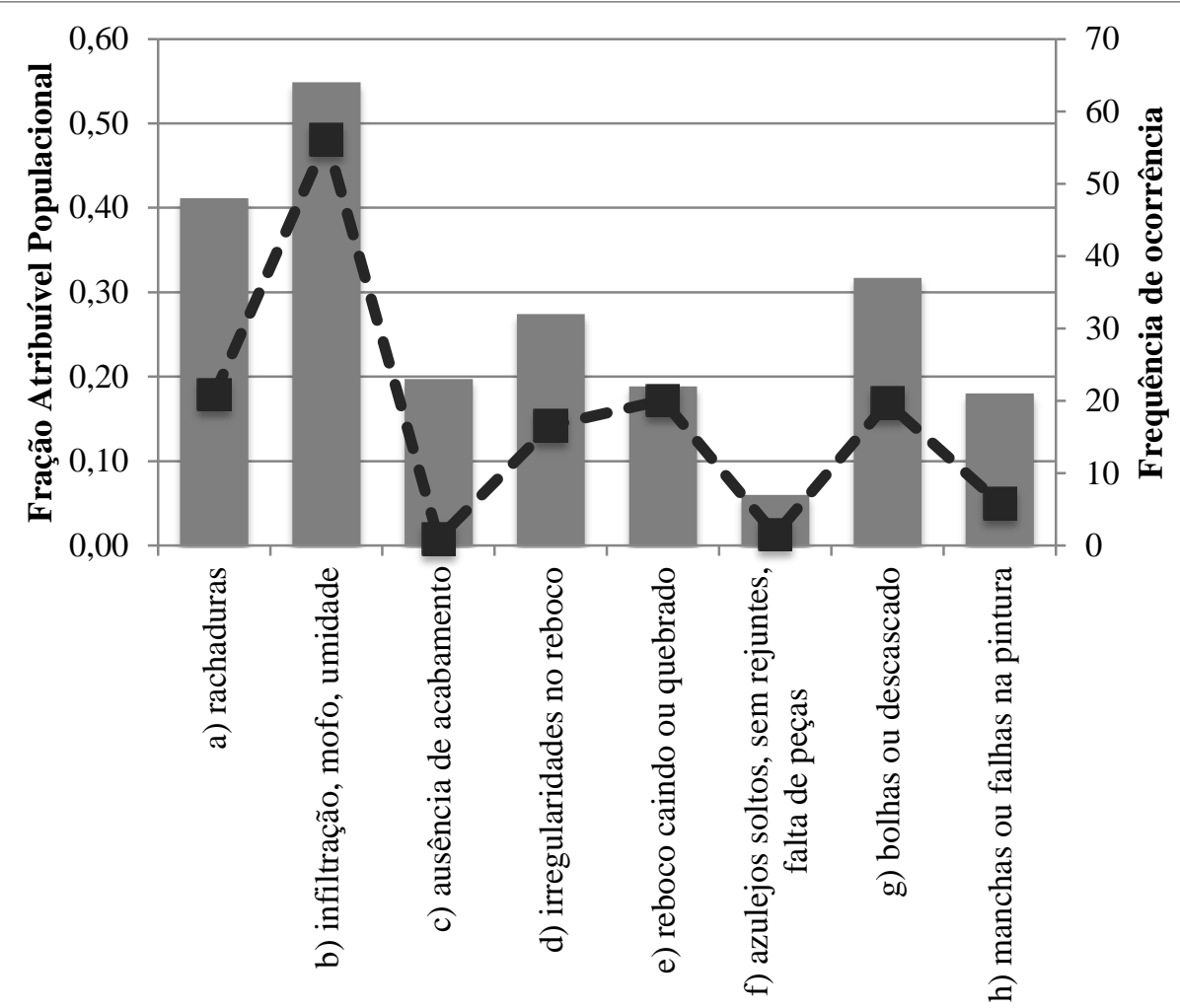

Itens do subsistema Vedações verticais/Paredes

Frequência de ocorrência ? Fração Atribuível Populacional

Tabela 5 - Dados utilizados no cálculo do indicador do subsistema vedações verticais/paredes

\begin{tabular}{lccc}
\hline & Proporções & FAP & Proporções*FAP \\
\hline Rachaduras nas paredes & 0,720 & 0,179 & 0,129 \\
Infiltração, mofo, umidade & 0,960 & 0,481 & 0,462 \\
Ausência de acabamento & 0,340 & 0,008 & 0,003 \\
Irregularidades no reboco & 0,480 & 0,142 & 0,068 \\
Reboco caindo ou quebrado & 0,340 & 0,172 & 0,058 \\
Azulejos soltos, sem rejuntes, faltam peças & 0,100 & 0,013 & 0,001 \\
Bolhas ou descascados & 0,550 & 0,169 & 0,093 \\
Manchas ou falhas na pintura & 0,310 & 0,050 & 0,016 \\
\hline Somatórios & & $\mathbf{1 , 2 1 4}$ & $\mathbf{0 , 8 3 0}$ \\
\hline
\end{tabular}

Tabela 6 - Indicadores por subsistema (IS) geral e para cada empreendimento estudado

\begin{tabular}{lllll}
\hline Indicador por subsistema & EHIS A & EHIS B & EHIS C & GERAL \\
\hline Paredes & 0,64 & 0,63 & 0,72 & 0,68 \\
Piso/Teto & 0,41 & 0,37 & 0,54 & 0,47 \\
Portas/Janelas & 0,61 & 0,45 & 0,50 & 0,51 \\
Instalações Elétricas & 0,25 & 0,22 & 0,16 & 0,20 \\
Instalações Hidrossanitárias & 0,25 & 0,23 & 0,42 & 0,34 \\
\hline
\end{tabular}

30 Berr, L. R.; Echeveste, M. E. S.; Lorenzi, L. S.; Formoso, C. T. 


\section{Indicador de falhas geral}

A combinação entre os indicadores por subsistema e o índice de gravidade percebida resultam no indicador de falhas geral por empreendimento $\left(I_{\text {Falhas }}\right)$, obtido pela Equação 4 . O primeiro avalia o efeito das falhas de cada subsistema sobre a qualidade da edificação na visão do usuário enquanto que o segundo incorpora a percepção de gravidade de cada subsistema, ou seja, como cada subsistemas impacta na qualidade da edificação.

A Tabela 7 apresenta o conjunto de métricas gerado neste estudo em sequência de cálculo para a formulação do indicador de falhas. Cada indicador por subsistema é multiplicado pela gravidade percebida, gerando o indicador por subsistema ponderado, somados e divididos pelo somatório da gravidade percebida. Na Equação 6 é apresentado o cálculo do indicador de falhas da amostra (geral).

Como exemplo de uso das informações, no empreendimento C os IS em "Piso e Teto" e "Portas e Janelas" têm valores semelhantes, mas o índice de gravidade percebida (GP) direciona para a necessidade de mais atenção ao primeiro, para buscar melhorias na percepção de qualidade do usuário. O mesmo ocorre na comparação do indicador ponderado em "paredes" e em "piso teto", em que o primeiro é amplificado, diferentemente do segundo, que mantém semelhança com sua escala original. Os indicadores de falhas de empreendimentos, por sua vez, permite a comparação entre estes, indicando o grau com que a percepção negativa dos usuários sobre cada subsistema afeta sua visão sobre a qualidade construtiva de cada empreendimento.

$I_{\text {Falhas }}=\frac{3.21}{6.00}=0,535$

Eq. 6

A Tabela 8 apresenta os resultados para os indicadores de falhas, geral e estratificado por empreendimento, bem como as médias das notas de qualidade atribuídas pelos entrevistados. Também são apresentadas taxas de reclamações das UH. Os indicadores gerais podem ser utilizados como valores de referência sobre uma única empresa construtora devido às delimitações proferidas à amostra. É possível perceber que os indicadores apresentam valores medianos e com certa similaridade entre empreendimentos.

Tabela 7 - Comparativo entre métricas propostas - estratificado por empreendimento e geral

\begin{tabular}{|c|c|c|c|c|c|c|c|}
\hline & & Paredes & $\begin{array}{c}\text { Piso e } \\
\text { Teto } \\
\end{array}$ & $\begin{array}{l}\text { Portas e } \\
\text { Janelas } \\
\end{array}$ & $\begin{array}{c}\text { Instalações } \\
\text { Elétricas }\end{array}$ & $\begin{array}{c}\text { Instalações } \\
\text { Hidrossanitárias } \\
\end{array}$ & IFalhas \\
\hline \multirow[t]{3}{*}{ EHIS A } & $\begin{array}{l}\text { Indicador por } \\
\text { subsistema (IS) }\end{array}$ & 0,640 & 0,410 & 0,610 & 0,250 & 0,250 & \multirow[b]{3}{*}{$\mathbf{0 , 5 4 0}$} \\
\hline & $\begin{array}{l}\text { Gravidade } \\
\text { percebida (GP) }\end{array}$ & 2,545 & 1,182 & 1,473 & 0,400 & 0,400 & \\
\hline & IS ponderado & 1,645 & 0,491 & 0,904 & 0,102 & 0,101 & \\
\hline \multirow[t]{3}{*}{ EHIS B } & $\begin{array}{l}\text { Indicador por } \\
\text { subsistema (IS) }\end{array}$ & 0,630 & 0,370 & 0,450 & 0,220 & 0,230 & \multirow[b]{3}{*}{0,495} \\
\hline & $\begin{array}{l}\text { Gravidade } \\
\text { percebida (GP) }\end{array}$ & 2,798 & 1,111 & 1,359 & 0,176 & 0,556 & \\
\hline & IS ponderado & 1,768 & 0,419 & 0,615 & 0,039 & 0,130 & \\
\hline \multirow[t]{3}{*}{ EHIS C } & $\begin{array}{l}\text { Indicador por } \\
\text { subsistema (IS) }\end{array}$ & 0,720 & 0,540 & 0,500 & 0,160 & 0,420 & \multirow[b]{3}{*}{0,565} \\
\hline & $\begin{array}{l}\text { Gravidade } \\
\text { percebida (GP) }\end{array}$ & 2,225 & 1,431 & 0,941 & 0,279 & 1,124 & \\
\hline & IS ponderado & 1,611 & 0,780 & 0,471 & 0,047 & 0,481 & \\
\hline \multirow[t]{3}{*}{ GERAL } & $\begin{array}{l}\text { Indicador por } \\
\text { subsistema (IS) }\end{array}$ & 0,680 & 0,470 & 0,510 & 0,200 & 0,340 & \multirow[b]{3}{*}{$\mathbf{0 , 5 3 5}$} \\
\hline & $\begin{array}{l}\text { Gravidade } \\
\text { percebida (IG) }\end{array}$ & 2,465 & 1,301 & 1,109 & 0,247 & 0,878 & \\
\hline & IS ponderado & 1,681 & 0,612 & 0,569 & 0,050 & 0,296 & \\
\hline
\end{tabular}

Tabela 8 - Comparativo entre índices - estratificado por empreendimento e geral

\begin{tabular}{lrrrc}
\hline & EHIS A & EHIS B & EHIS C & GERAL \\
\hline Indicador de falhas & 0,54 & 0,49 & 0,56 & 0,53 \\
Média da nota da qualidade & 0,64 & 0,68 & 0,59 & 0,63 \\
\% de UH que reclamaram & 0,29 & 0,39 & 0,47 & 0,39 \\
Reclamações/UH & 0,55 & 0,88 & 1,12 & 0,85 \\
\hline
\end{tabular}




\section{Checagem do indicador}

Para verificar a adequação do índice, calculou-se a correlação entre a nota média da qualidade atribuída no questionário e o indicador de falhas obtido na Equação 4. O coeficiente de correlação de Pearson apresentou resultado alto e negativo ( $\mathrm{r}=-0,996$, $\mathrm{p}$-valor $<0,001)$, indicando que, quanto maior o indicador de falhas, menor a nota média da qualidade obtida. Contudo, não se verifica a mesma associação com as demais métricas analisadas e apresentadas na Figura 3, a saber: a porcentagem de UH reclamantes e a média de reclamações por unidade habitacional, por empreendimento e geral.

É possível observar que no EHIS C, apesar da maior quantidade de reclamações, o indicador de falhas não apresenta diferença em relação à nota de qualidade. No empreendimento A a situação é diferente, pois, apesar de apresentar a menor taxa de reclamações $(0,56)$ - aproximadamente 1 reclamação a cada 2 habitações -, o indicador geral de falhas apresentou resultado de 0,54. Esta, por ser uma medida mais agregada, pode ser entendida como percepção negativa da confiabilidade do empreendimento. Uma explicação pertinente é que as falhas existentes no empreendimento A impactam mais fortemente na percepção de qualidade dele. Já o EHIS B tem o menor indicador de falhas e a maior média das notas de qualidade, ambos na percepção dos usuários, indicando tendência de melhor qualidade sob os aspectos percebidos pelos usuários que nos outros empreendimentos.

\section{Conclusões}

O objetivo deste trabalho foi propor um instrumento para mensurar a qualidade do produto com base na percepção do usuário final das habitações de interesse social, cuja resposta é apresentada na forma de um indicador de falhas. $\mathrm{O}$ indicador proposto busca mensurar as falhas da edificação considerando a gravidade da ocorrência dessas falhas, possibilitando avaliar a confiabilidade e a qualidade percebida. A estrutura do instrumento de avaliação possibilita identificar o impacto de falhas construtivas no desempenho da habitação ao longo do tempo sob a perspectiva do usuário, entendida neste artigo como a qualidade percebida. $\mathrm{O}$ uso de um indicador de falhas com enfoque na percepção dos usuários possibilita avaliar o desempenho das empresas construtoras em atender aos requisitos dos clientes, além de identificar os subsistemas que mais influenciam na percepção de qualidade dos usuários em cada uma das empresas. Assim, é possível priorizar processos que devem ser monitorados com mais ênfase, a fim de contribuir para a melhoria da qualidade e para o desempenho das HIS

Tendo em vista que o indicador é de fácil obtenção, pode ser incluído às rotinas das empresas construtoras que possuem sistema de gestão da qualidade (SGQ), sendo útil para monitorar as necessidades dos clientes. Em uma abordagem estratégica, tanto das empresas construtoras quanto de agentes de políticas públicas na área habitacional, esse indicador pode ser útil para o desenvolvimento de futuros empreendimentos, bem como contribuir para a formação de um sistema de benchmarking para o segmento de HIS.

Figura 3 - Comparação das métricas empregadas no estudo - estratificado por empreendimento e geral

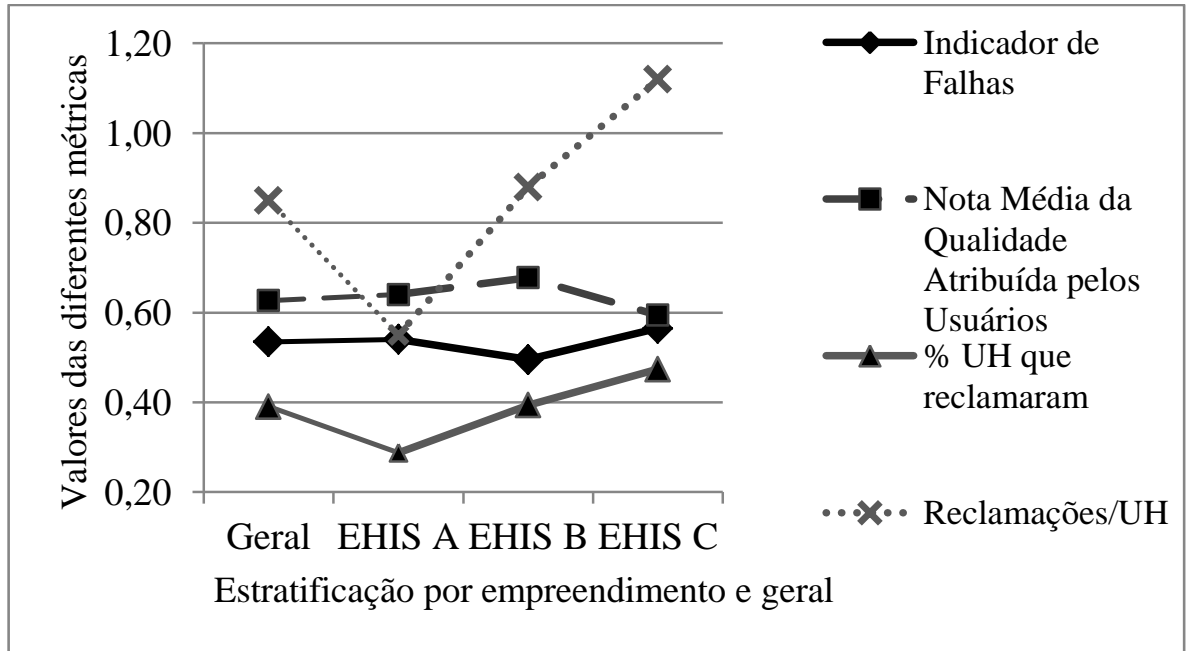

32 Berr, L. R.; Echeveste, M. E. S.; Lorenzi, L. S.; Formoso, C. T. 
Já os indicadores por subsistema possibilitam decisões táticas e operacionais específicas para cada uma das partes da edificação, mas alinhadas com a visão do usuário. $O$ enfoque dado na pesquisa proporciona a vinculação direta das falhas observadas pelos usuários a aspectos tratados nas definições de projeto, como decisões dos sistemas e métodos construtivos que serão empregados nos empreendimentos futuros. Também auxilia em decisões da etapa de produção e no controle da qualidade dos serviços, possibilitando a priorização de ações de melhoria da qualidade sobre os subsistemas que mais impactam na percepção de qualidade dos usuários.

No que se refere a diferenças de comportamento entre a frequência de ocorrência e a FAP contendo a percepção do usuário, esta análise indica que a simples identificação da maior ocorrência de um problema não sinaliza diretamente a percepção do usuário e evidencia a utilidade da fração atribuível para a priorização de ações de melhoria e também corretivas.

Por fim, cabe salientar a forma como o morador percebe as falhas de sua habitação. Durante a coleta de dados, mesmo quando a equipe técnica observava determinada falha, em alguns casos o entrevistado não a percebia. Nesse sentido, entende-se que em trabalhos futuros a avaliação das habitações de maneira combinada entre a visão interna das empresas normalmente traduzida pela visão do técnico e a visão externa do morador pode trazer contribuiçõos para o entendimento da qualidade das habitações.

\section{Referências}

ABIKO, A. K.; ORNSTEIN, S.W. Inserção Urbana e Avaliação Pós-Ocupação (APO) da Habitação de Interesse Social. São Paulo: FAUUSP, 2002. (Coletânea Habitare/FINEP, 1) acessado em 24/04/2012

http://www.habitare.org.br/pdf/publicacoes/arquiv os/79.pdf.

ALEXANDRE, I. F. Manifestações patológicas em empreendimentos habitacionais de baixa renda executados em alvenaria estrutural: uma análise da relação de causa e efeito. Porto Alegre, 2008. Dissertação (Mestrado em Engenharia Civil) - Escola de Engenharia, Universidade Federal do Rio Grande do Sul, Porto Alegre, 2008.
ANDRADE, J.; DAL MOLIN, D. C. C

Durabilidade das Estruturas de Concreto Armado: análise dos elementos estruturais mais degradados no estado de Pernambuco. In: ENCONTRO

NACIONAL DA TECNOLOGIA DO AMBIENTE CONSTRUÍDO: QUALIDADE DO PROCESSO CONSTRUTIVO, 7., Florianópolis, 1998. Anais... Florianópolis, 1998.

ARDITI, D.; GUNAYDIN, H. M. Total Quality Management in the Construction Process. International Journal of Project Management, v. 15, n. 4, p. 235-243, 1997.

ASSOCIAÇÃO BRASILEIRA DE NORMAS TÉCNICAS. NBR 15575: edifícios habitacionais: desempenho: parte 1: requisitos gerais. Rio de Janeiro, 2013.

\section{ASSOCIAÇÃO BRASILEIRA DE NORMAS}

TÉCNICAS. NBR ISO 9001: sistema de gestão da qualidade: requisitos. Rio de Janeiro, 2008.

BARTZ, C. F. Identificação de melhorias no processo de controle da qualidade em empreendimentos habitacionais de baixa renda. Porto Alegre, 2007. Dissertação (Mestrado em Engenharia Civil) - Escola de Engenharia, Universidade Federal do Rio Grande do Sul, Porto Alegre, 2007.

BERR, L. R.; FORMOSO, C. T. Método Para Avaliação da Qualidade de Processos Construtivos em Empreendimentos Habitacionais de Interesse Social. Ambiente Construído, Porto Alegre, v. 12, n. 2, p. 77-96, jun./ago. 2012.

BLACHÈRE, G. Savoir Bâtir: habitabilité, durabilité, économie des bâtiments. Paris, 1969. Disponível em:

<http://babel.hathitrust.org/cgi/pt?id=uc1.b432152 5>. Acesso em: 11 ago. 2015.

BONATTO, F. S. et al. O uso da percepção do usuário na avaliação da qualidade da habitação de interesse social: método de coleta, processamento e análise. In: ENCUENTRO LATINOAMERICANO DE GESTIÓN Y ECONOMIA DE LA CONSTRUCCION, 3., Santiago, 2009. Anais... Santiago: Universidad de los Andes, 2009.

BORGES, C. A. O conceito de desempenho de edificações e a sua importância para o setor da construção civil no Brasil. São Paulo, 2008. Dissertação (Mestrado em Engenharia Civil) Escola Politécnica, Universidade de São Paulo, 2008. 
CARDOSO, F. F. Certificações "setoriais" da qualidade e microempresas: o caso das empresas especializadas de construção civil. São Paulo, 2003. Tese (Livre Docência) - Escola Politécnica, Universidade de São Paulo, 2003.

FIESS, J. R. et al. Causas da Ocorrência de Manifestações Patológicas em Conjuntos Habitacionais do Estado de São Paulo. In: CONFERÊNCIA LATINO-AMERICANA DE CONSTRUÇÃO SUSTENTÁVEL; ENCONTRO NACIONAL DE TECNOLOGIA DO AMBIENTE CONSTRUÍDO, 10., São Paulo, 2004. Anais... São Paulo: ANTAC, 2004.

GARVIN, D. A. Competing on the Eight Dimensions of Quality. Harvard Business Review, November-D, 1987.

GARVIN, D. A. Gerenciando a qualidade: a visão estratégica e competitiva. Rio de Janeiro: QualityMark, 2002.

HANLEY, J. A. A Heuristic Approach to the Formulas For Population Attributable Fraction. Journal of epidemiology and community health, v. 55, n. 7, p. 508-514, 2001.

HELMAN, H.; ANDERY, P.R.P. Análise de falhas (aplicação dos métodos de FMEA-FTA). Belo Horizonte. MG: Fundação Christiano Ottoni, Escola de Engenharia da UFMG, 1995.

HOPP, W. J.; SPEARMAN, M. L. Factory Physics: foundation of manufacturing management. New York: McGraw Hill, 2000.

INTERNATIONAL ORGANIZATION FOR STANDARDIZATION. ISO6241. Performance satandards in building - Principles for their preparation and factor to be considered. London, 1984.

JURAN, J. M. Juran on planning for quality. The Free Press, New York, 1988.

\section{KOSKELA, L. Application of the New} Production Philosophy to

ConstructionTechnology. Stanford University Center for Integrated Facility Engineering, 1992. (CIFE Technical Report, n. 72).

KOWALTOWSKI, D. C. C. K. et al. SitePlanning Guidelines for Low Income Housing in the State of São Paulo, Brazil, Based on POE and Axioms. In: CONFERENCE OF THE INTERNATIONAL ASSOCIATION FOR PEOPLE-ENVIRONMENT STUDIES, 18. Vienna, 2004. Anais... Vienna, 2004.
LORENZI, L. S. Análise crítica e proposições de avanço nas metodologias de ensaios experimentais de desempenho à luz da $\mathrm{ABNT}$ NBR 15575 (2013) para edificações habitacionais de interesse social térreas. Porto Alegre, 2013. Tese (Doutorado em Engenharia Civil) - Escola de Engenharia, Universidade Federal do Rio Grande do Sul, Porto Alegre, 2013.

MILLS, A., LOVE, P. E., WILLIAMS, P. Defect Costs in Residential Construction. Journal of Construction Engineering and Management . v.125, n.1, p. 12-16, 2009.

MITIDIERI FILHO, C. V. Qualidade e Desempenho na Construção Civil. In: ISAIA, G. C. (Org.). Materiais de Construção Civil e os Princípios de Ciência e Engenharia dos Materiais. São Paulo: IBRACON, 2007. v. 1.

MITIDIERI, C. V.; HELENE, P. R. Avaliação de desempenho de componentes e elementos construtivos inovadores destinados a habitação: proposições específicas à avaliação do desempenho estrutural. São Paulo, 1998.

PARASURAMAN, A. Reflections on Gaining Competitive Advantage Through Customer Value. Journal of the Academy of Marketing Science, v. 25, n. 2, p. 154-161, mar. 1997.

RAUSAND, M.; KNUT, O. The Basic Concepts of Failure Analysis. Reliability Engineering and System Safety, v. 53, n. 1, p. 73-83, 1996.

RICHTER, C. Qualidade da alvenaria estrutural em habitações de baixa renda: uma análise da confiabilidade e da conformidade. Porto Alegre, 2007. Dissertação (Mestrado em Engenharia Civil) - Escola de Engenharia, Universidade Federal do Rio Grande do Sul, Porto Alegre, 2007.

SAVAGE, G. J.; CARR, S. M. Interrelating Quality and Reliability in Engineering Systems. Information Systems, v. 14, n. 1, p. 137-152, 2001.

SILVA, M. A. C. Dilemas da Construção. Revista da Construção. 2010, n 92.STONEROMERO, E. F.; STONE, D. L.; GREWAL, D. Development of a Multidimensional Measure of Perceived product quality. Journal of Quality Management, v. 2, n. 1, p. 87-111, jan. 1997.

WANKHADE, L.; DABADE, B. M. TQM With Quality Perception: a system dynamics approach. The TQM Magazine, v. 18, n. 4, p. 341-357, 2006.

WOODRUFF, R. B. Customer Value: the next source for competitive advantage. Jornal of Academy of Marketing Science, v. 25, n. 2, p. 135-153, 1997. 
WU, S. et al. Reliability in the Whole Life Cycle of Building Systems. Engineering, Construction and Architectural Management, v. 13, n. 2, p. 136-153, 2006.

ZEITHAML, V. A. Consumer Perceptions of Price, Quality, and Value: a means-end model and synthesis of evidence. The Journal of Marketing, v. 52, n. 3 , p. $2-22,1988$.
ZHANG, Q. Quality Dimensions, Perspectives and Practices a Mapping analysis. International Journal of Quality \& Reliability Management, v. 18, n. 7, p. 708-721, 2001.

\section{Letícia Ramos Berr}

Escola de Engenharia | Universidade Federal do Rio Grande do Sul | Av. Osvaldo Aranha, 99, $3^{\circ}$ andar, Centro | Porto Alegre - RS - Brasil | CEP 90035-190 | Tel.: (51) 3308-3518 | E-mail: leticia.berr@ufrgs.br

\section{Márcia Elisa Soares Echeveste}

Departamento de Estatística, Instituto de Matemática | Universidade Federal do Rio Grande do Sul | Tel.: (51) 3308-4297 |

E-mail: echeveste@producao.ufrgs.br

\section{Luciani Somensi Lorenzi}

Pós-Graduação em Engenharia Civil | Universidade Federal do Rio Grande do Sul | E-mail: luciani.lorenzi@gmail.com

\section{Carlos Torres Formoso}

Pós-Graduação em Engenharia Civil | Universidade Federal do Rio Grande do Sul | Tel.: (51) 3308-3518 | E-mail: formoso@ufrgs.br

Revista Ambiente Construído

Associação Nacional de Tecnologia do Ambiente Construído

Av. Osvaldo Aranha, $99-3^{\circ}$ andar, Centro

Porto Alegre - RS - Brasil

$$
\text { CEP } 90035-190
$$

Telefone: +55 (51) 3308-4084

Fax: +55 (51) 3308-4054

www.seer.ufrgs.br/ambienteconstruido

E-mail: ambienteconstruido@ufrgs.br 